\title{
SUPERVISION AND CONTROL SYSTEM OF METROPOLITAN SCOPE BASED ON PUBLIC COMMUNICATION NETWORKS
}

\author{
Sempere, V. ${ }^{*}$ Albero, T. ${ }^{*}$ Silvestre, J. ${ }^{* *}$ \\ * Dpto. Communications. UPV. \\ ${ }^{* *}$ Dpto. Computer Engineering. UPV.
}

\begin{abstract}
The present systems of control based on polling by radio frequency must evolve to be able to carry advanced telecommunication services, such as capture and multimedia information management as well as control information. In this paper a supervision and control system based in public networks that complements a classic polling system allowing to incorporate new information services and eliminating the typical long delays of these kinds of systems is described. Two alternatives of communication are evaluated, point to point connections and Virtual Private Networks, VPNs/IP on ISDN, for the interconnection of the stations and the control center. For this, images are transmitted as well as the control information through both types of connection, the throughput of the transmission is evaluated and its behavior is observed. Compression techniques are used and the different parameters that can influence the compressed image quality are analyzed. The viability of this type of solution in the proposed scenarios is demonstrated. Copyright (C)2003 IFAC
\end{abstract}

Keywords: Images compression, Communication networks, Supervision, Control system.

\section{INTRODUCTION}

In this paper a newly designed system is introduced to modernize the control and supervision of a large city purification network. The form in which it operates is over public communication networks and it is able to manage control and multimedia information centrally. It is designed to be installed independently, or jointly with existing control systems since it is able to interchange control information directly from the installation sensors and actuators or point to point from any command equipment (PLC, terminal, etc), to capture images of critical areas of the installation selected by the operator and to transmit them to

1 supported by UPV (Polytechnic University of Valencia. Spain) a central station where they are processed in real time.

The purification networks are normally governed by installations distributed of metropolitan scope based on PLCs that use polling (Ibe and Trivedi, 1990; Haverkort, 1999) by means of radio frequency for the communications between the central station that houses a SCADA (Luque et $a l ., 1996)$ and each and every one of the remote nodes. This type of solution supposes a bottleneck during the period on which new services and applications are being implemented. The reasons that have suggested these actions are:

The installation is constantly growing and the procedure of polling cannot offer the time guarantees that are required, since to greater number 


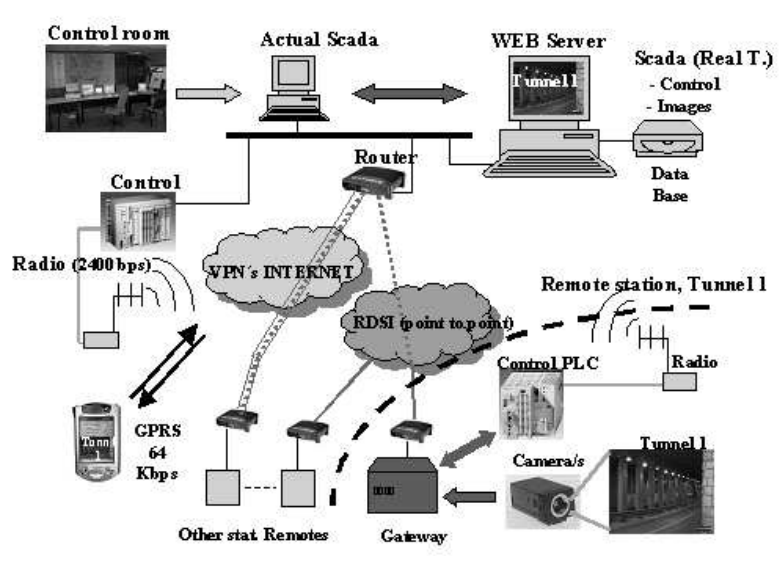

Fig. 1. System general scheme

of stations to be consulted by the central station, greater is the time lapse between successive consultations. The new system has to handle the later incorporation of new remote stations without any time increase.

It is necessary to equip the system with redundant communications to increase their reliability, implementing new advanced monitoring and control services that facilitate both, the inspection of any critical zone within the installation and the remote teleoperation The communications have to be protected against any possible unwanted third party interference.

In this paper a global description of the architecture system is presented, followed by a detailed evaluation of the processing, transmission and storage of the images. The performance of the system is analyzed, obtaining the transmission rate of images by means of two public networks communication alternatives:

(1) VPN/IP (Virtual Private Network) (Kosiur, 1998)

(2) point to point connection.

The paper is structured in the following way: In section 2 the different parts of the new system are described and the chosen solution is justified. In section 3 the treatment process for the transmission images is described. Later in section 4 , the method of storing and accessing the information is described. Finally, the developed application and some basic measurements which demonstrate the viability of the proposed solutions are shown.

\section{SYSTEM COMPONENTS}

It is made up of three fundamental parts (see Fig. 1):

- Central station.

- Remote Nodes/gateways.

- Communication network.

\subsection{Central Station}

This is the part charged with managing the information coming from all the installation and controlling the remote communication between the central and remote stations. It communicates simultaneously (using several connections TCP/IP) with all the remote stations, eliminating the slow cycles of polling and contributing in joint installation cases with remote control systems, a redundant communication channel which equips the installation as a whole with a high degree of reliability and availability.

The central station uses equipment which does the SCADA in the new network and that houses the states and images data base (where it is used like a SQL Server 7.0. motor). A background process is charged with preserving coherence between the old data base (updated by polling) and the new one. The central station also houses a Web server which permits the data base which registers the information of each remote to be consulted locally and through the Internet.

By means of the local processing in the central station (developed with Borland C++ Builder 5), it allows the visualization of images coming from one or more installations simultaneously, as well as obtaining control data, greatly facilitating the tasks of tracking incidences.

The Web application has been developed with Javascript, VBScript, ASP and HTML. It is possible to make the Web connection from fixed or movable nodes using GPRS (Hoymann and Stuckmann, 2002), as a Web page has been designed that at the moment of connection detects and adapts to the type of user fixed (PC) or movable (PDA, Compaq iPAQ 3970 has been used). It is important to emphasize that for security reasons the Web usuary can only consult information and does not have control of the system. If the client connects itself from a PC, it is allowed to see data control and images in real time, furthermore the stored images. However if it accedes through a PDA it can see realtime images as well as the information of the states.

In Fig.2 the block architecture of the central station can be seen, where the arrows indicate the incoming/outgoing flow of information.

There are three types of subprocesses existing in the local processing in the central station as well as in the remote processing node; those executed at the request of the user (manual), those that have previously defined parameters and offer information in periodic form, and those that are not predictable and notify of some change of aperiodic form. 


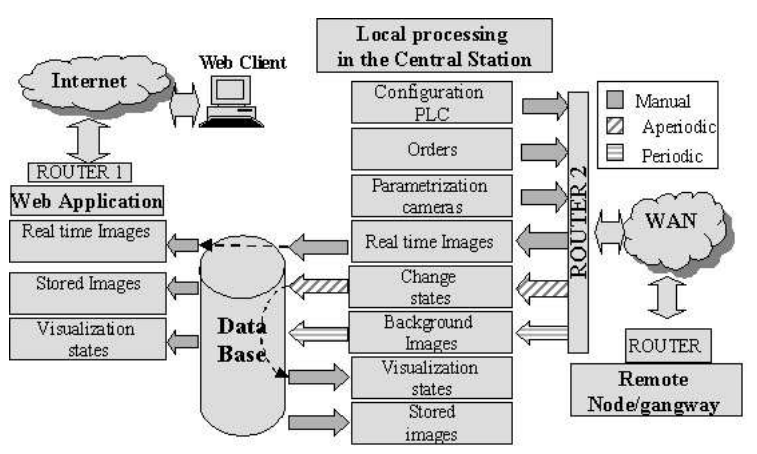

Fig. 2. Block architecture of the central station

\subsection{Remote Node/Gateway}

This is the equipment located in each of the remote stations to be governed. It is the gateway between the communication network and the local control system of each remote station. It is constituted of an industrial micro PC with operating system W-2000 Professional and a powerful applications developed with Borland $\mathrm{C}++$ Builder 5 and Visual Microsoft $\mathrm{C}++$ which makes easy adaptation to any installation.

It can also capture information coming from cameras (up to 4) located in the installation, process it and jointly transmit it to the central station by the communication language available in each case. It is able to communicate with any PLC on the market (the protocol of Simatic S5/S7 has been used) bidirectionally and to map in a simple way the main variables of the process. The remote processing node determines when susceptible variables being sent to the central station have taken place and executes an automatic update process.

The parametrization of the remote Node/Gateway is done centrally through the central station. The information provided is as follows:

- Variables to read/write from the PLC.

- Resolution, quality and rate of compression of the images.

- Position of the cameras.

- Intervals of capture of the images.

In Fig.3 the block architecture of the remote gateway can be seen, where as in the central station the arrows indicate the information flow.

\subsection{Communication network}

This has to give communication support in concurrent form to all the remote stations, allowing the incorporation of new services in real time such as the transport of images, states and orders. The present system of polling by means of radio frequency is a redundant system acting as backup to operate in case of failure of minimum services.

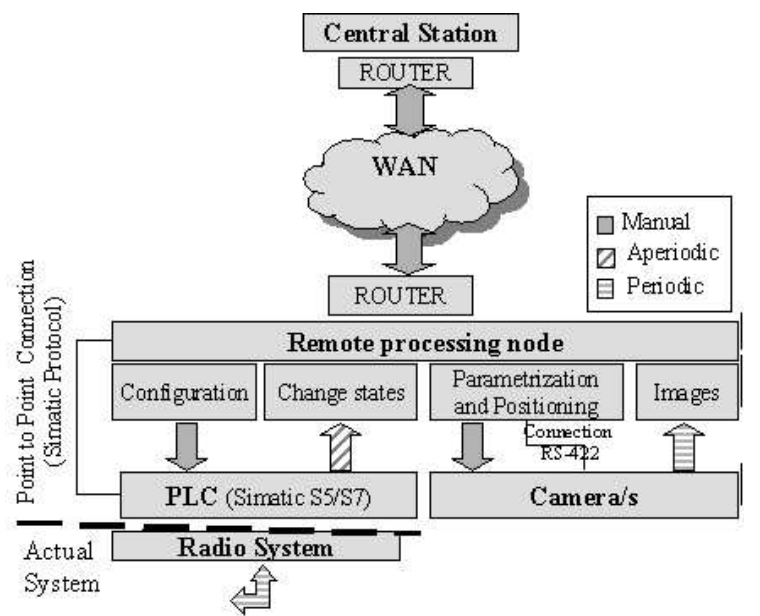

Fig. 3. Block architecture of the remote station

After a detailed study of the diverse possibilities two possible alternatives for the interconnection of the stations have been studied. These are:

- Use of point to point connections between the central station and each of the remote stations through ISDN (Integrated Services Digital Network)

- VPN (Virtual Private Network) through Internet with ISDN access.

In the result section a comparison between the two is made.

The criteria for determining this choice are as follows, in the first place, the adjustment of the service offered by the network (guaranteed bandwidth, asymmetry absence), secondly the area of coverage in urban zones. Other alternatives such as HFC (Hybrid Fiber Coax) or ADSL have not been considered in this paper, but they will be reason for study in later papers.

The central station has a ISDN router with sufficient basic accesses $(2 \mathrm{~B}+\mathrm{D})$ or a primary one $(30 \mathrm{~B}+\mathrm{D})$ according to the needs. The traffic between the central equipment and the remote stations is guided through this. A local area network infrastructure carries the communication between the new central station, the router, the old central station and the central PLC responsible for the polling tasks. At each remote station a basic access ISDN arrives. In the central station the control information and the images are simultaneously collected from all the remote stations.

When the communication channel is available, all exchange of information between the central station and the remote ones is made by means of TCP/IP, facilitating the incorporation of new services and the movement toward other futures possibilities with QoS (Quality of Service) on Internet (Ferguson and Huston, 1998). 


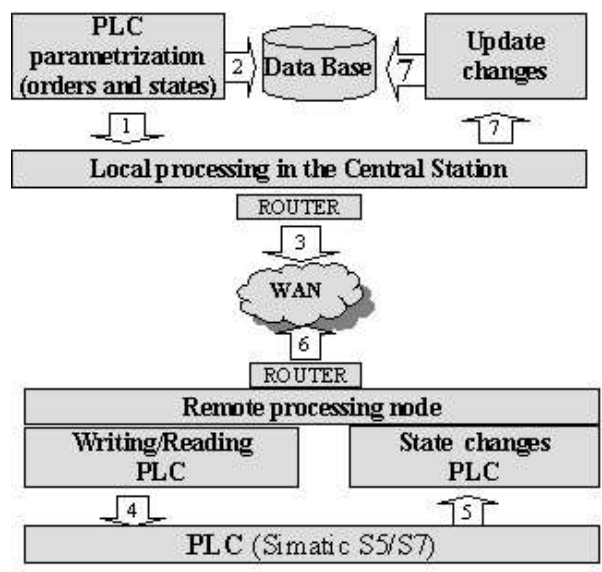

Fig. 4. Protocol for the control data processing

\section{CONTROL INFORMATION TREATMENT}

In Fig.4 the processes that take part in the control data processing can be seen, furthermore the arrows are also numbered to indicate the order of execution.

The central station does the variables parametrization(1) to read (states configuration ) and to write (orders configuration) from the PLC. In this configuration, the position in the memory zone (for the Simatic PLC, DB (Data Block), DW (Data Word) and bit of that word ) or the mark zone, (MB (Mark Block), MW (Mark Word), and bit) that contains the state/order information, is indicated. When the parametrization in the central station is finalized, the configuration is stored in the data(2) base and this parametrization is immediately sent(3) to the remote station, which by means of the corresponding process establishes the memory or mark zone and does the state reading(4) in the PLC continuously. Also from the central station orders can be generated(3) to the remote station, by means of this action the writing(4) is done in the PLC.

The remote station communication with the PLC is made by means of the PlcS5 ActiveX Control, that uses the Siemens Simatic S5 AS511 protocol by a serial connection. This control, is able to detect any changes(5) that can happen in the PLC (the change can be caused by an incidence in the system or by an order executed from the central station). When this happens, from the remote station the alarm is sent(6) to the central station, then the appropriate measures can be taken. Furthermore, the state change is stored in the central station data base(7).

The control information travels in IP packages independently of the images. As the state variability is not elevated and only the changes are transmitted, this traffic does not affect the system throughput.

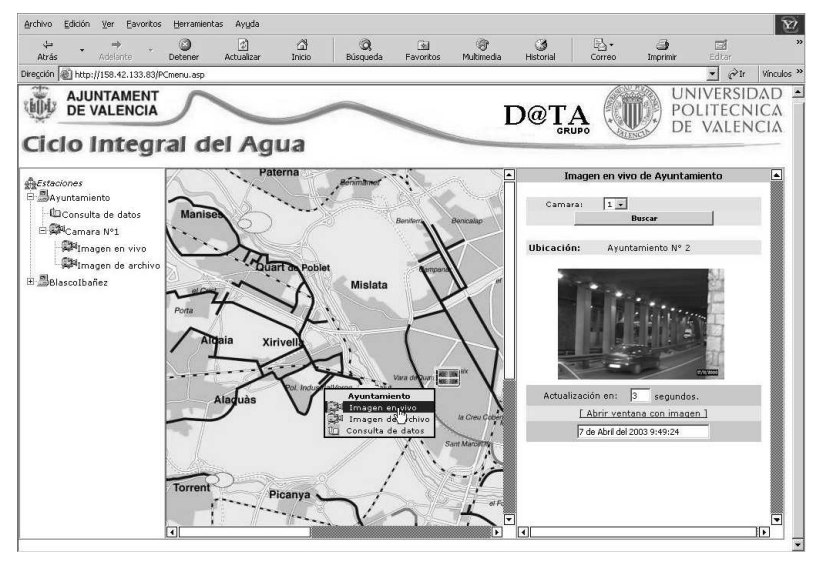

Fig. 5. Visualization of realtime images from the application Web

\section{IMAGE TREATMENT AND TRANSMISSION}

Images are transmitted compressed from the remote stations to the central station as this helps to reduce the bandwidth consumed by the transport of images although at the expense of image quality.

Due to the low speed of the transmission channel $(64 \mathrm{~Kb} / \mathrm{s})$, streaming of video is very difficult (Woods, 2002). Since it is an application of monitoring, where a great number of images per second is not necessary, JPEG2000 has been chosen for the compression which has a very good quality/compression relationship compared with JPEG.

Furthermore, codec has been chosen based on values obtained in the following parameters:

- Quality.

- Necessary/available bandwidth.

- Compression/decompression time.

After studying different codecs which are not going to be detailed in this paper, due to the good results given, the Morgan Multimedia MJPEG2000 is used.

The transmission/reception of images can be required by a local user in the central station or by a usuary Web (see Fig.5), although the local processing in the central station will be the one that demands and receives the images by means of the protocol that is outlined next. In Fig.6 the transmission/reception protocol can be seen in schematized form. The arrows indicate if it is dealing with traffic of communication between the processes (handshaking) or data. These are also numbered to indicate the order of execution. The remote station has two processes "CapturaImag" and "ClienteImag", the first is the one charged with capturing the camera images and to compress them (Morgan M-JPEG2000), also acting as a server. "ClienteImag" is the client and is 


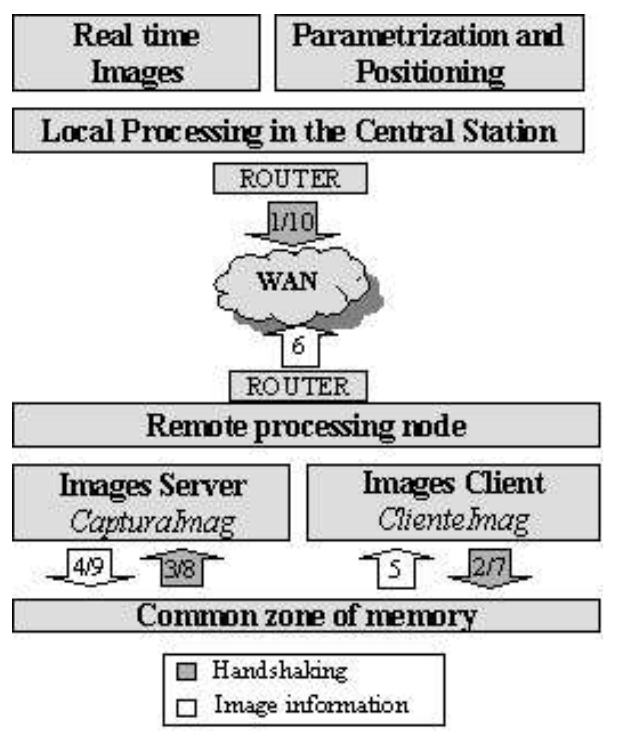

Fig. 6. Protocol for transmission of images

charged with transmission of images to the central station and also to receive the parametrization and positioning information. Both processes share a common zone of memory that allows them to write or to read information in synchronous form.

The central station is that which makes the parametrization and indicates when the transmission/reception can begin by means of message(1) to the "ClienteImag" process. When "ClienteImag" receives this message, it informs $(2,3)$ "CapturaImag" that a compressed image can be collocated(4) in the common memory zone when it is available. From the common memory zone "ClienteImag" takes $(5)$ the image and transmit(6) it to the process of the central station. When the complete image has been transmitted, it informs $(7,8)$ "CapturaImag" that it can again put(9) a new one in the memory zone. It is also indicated from the central station when the transmission/reception of the images must conclude(10) by means of another message to the "ClienteImag" process.

\section{IMAGE STORAGE AND RECOVERY}

The parameters of images storage are defined from the central station, differentiating into two types: a) storage in background, b) manual storage.

The first type is independent of the current situation, the parametrization has been done previously and the image capture is done with constant periodicity throughout the day. Manual storage is done at any moment when the situation could be critical (strong rain). If the operator is visualizing these images on the screen and considers it necessary, he can store these images in the data base for later study.

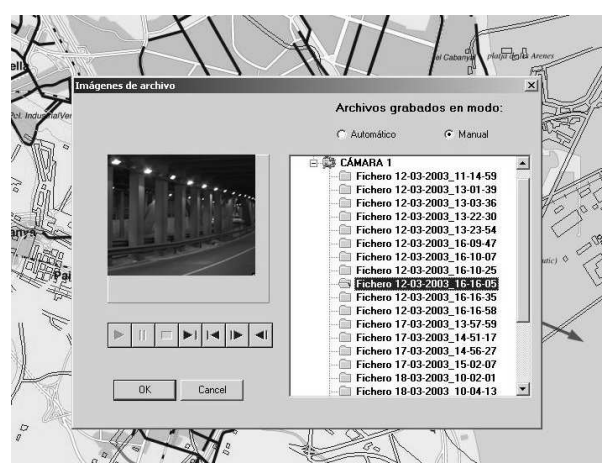

Fig. 7. Access to images stored in the central station

Table 1. Test Sequences

\begin{tabular}{lll}
\hline & Size(KB) & Duration(min) \\
\hline C2 & 341.192 & 2.34 \\
C3 & 364.450 & 2.36 \\
C4 & 472.302 & 3.16 \\
\hline
\end{tabular}

Table 2. Result of quality parameters after compression of sequences

\begin{tabular}{lllll}
\hline & PSNR (dB) & Size (bytes) & CT (s) & DT $(s)$ \\
\hline C2-FQ87 & 40.12 & 8051.41 & 0.158 & $1.13 \mathrm{E}-5$ \\
C3-FQ87 & 39.70 & 8317.10 & 0.108 & $1.09 \mathrm{E}-5$ \\
C4-FQ87 & 40.34 & 8292.52 & 0.110 & $1.13 \mathrm{E}-5$ \\
\hline C2-FQ56 & 37.67 & 5334.26 & 0.151 & $1.13 \mathrm{E}-5$ \\
C3-FQ56 & 37.19 & 5323.36 & 0.099 & $1.11 \mathrm{E}-5$ \\
C4-FQ56 & 38.00 & 5444.46 & 0.102 & $1.16 \mathrm{E}-5$ \\
\hline C2-FQ30 & 34.26 & 2849.93 & 0.146 & $1.12 \mathrm{E}-5$ \\
C3-FQ30 & 34.00 & 2851.76 & 0.151 & $1.09 \mathrm{E}-5$ \\
C4-FQ30 & 34.90 & 2861.08 & 0.096 & $1.12 \mathrm{E}-5$ \\
\hline C2-FQ18 & 31.77 & 1708.01 & 0.144 & $1.09 \mathrm{E}-5$ \\
C3-FQ18 & 31.57 & 1703.14 & 0.148 & $1.09 \mathrm{E}-5$ \\
C4-FQ18 & 32.48 & 1710.79 & 0.093 & $1.16 \mathrm{E}-5$ \\
\hline
\end{tabular}

For the capture of these images it is necessary to select: camera, quality, resolution and length of recording (maximum one hour). The storage is completed once the established time has passed or when the operator deems it correct to do so.

Later, the stored images can be recovered (background or manual) to be viewed and analyzed as can be seen in Fig.7.

\section{EXPERIMENTS AND RESULTS}

The tests to ascertain the efficiency of the system have been made with three video sequences stored on disc (see table 1) and not with real time images, to thus analyze a same test set. For ISDN communications, a single channel of $64 \mathrm{~Kb} / \mathrm{s}$ has been used.

In order to limit the number of tests two important parameters have been used: Forced Quality (FQ) and Constant Bit Rate (CBR) which influence the compression quality (PSNR), the size, the compression time (CT) and the decompression time (DT) of each image decisively. As can be seen in table 2 , for each sequence three quality levels 


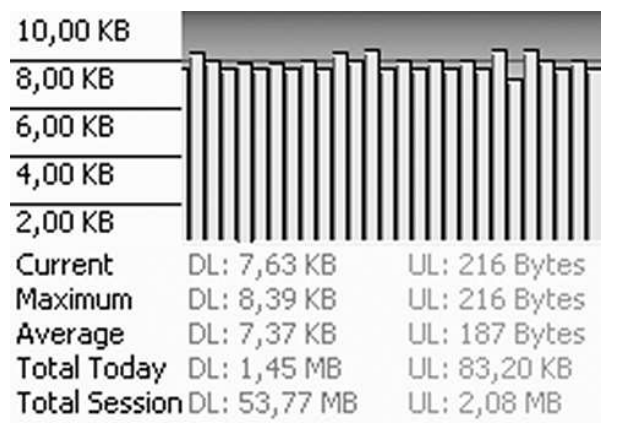

Fig. 8. Monitoring of the connection through ISDN

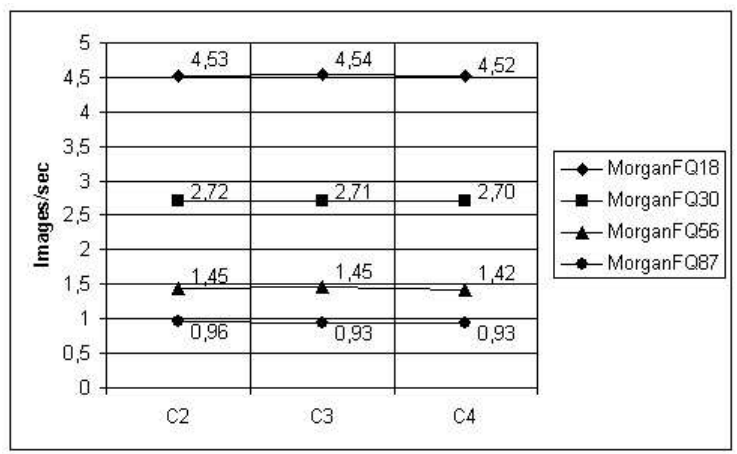

Fig. 9. Images/sec in the point to point connection, for different levels of compression

are going to be used. These have been obtained with different values from FQ (87, 56, 30 and 18). The compression and decompression time is insignificant against the time it takes to transmit an image considering that it has a theoretical speed of $64 \mathrm{~Kb} / \mathrm{s}$, and so is not considered in the calculations.

\subsection{Point to point connection through ISDN}

Diverse measurements have been taken in different hour strips, taking samples every two seconds and it has been observed that the obtained speed values of the channel are practically constant, with very little variation and without cuts (see Fig.8). For this reason an average value has been taken to calculate the number of images.

It is evident (see Fig.9) that with a greater compression, a greater amount of images can be sent with a cost of quality loss, but is necessary to consider that it is a monitoring application, where a high quality is not necessary. Therefore, it is necessary to reach a compromise between quality and compression level. After impartial evaluation by various different observers, one conclusion has been reached, the MorganFQ30 compression (PSNR of $34,38 \mathrm{~dB}$ ) offers an acceptable quality for a monitoring application and control, in which almost three images/sec. can be transmitted.

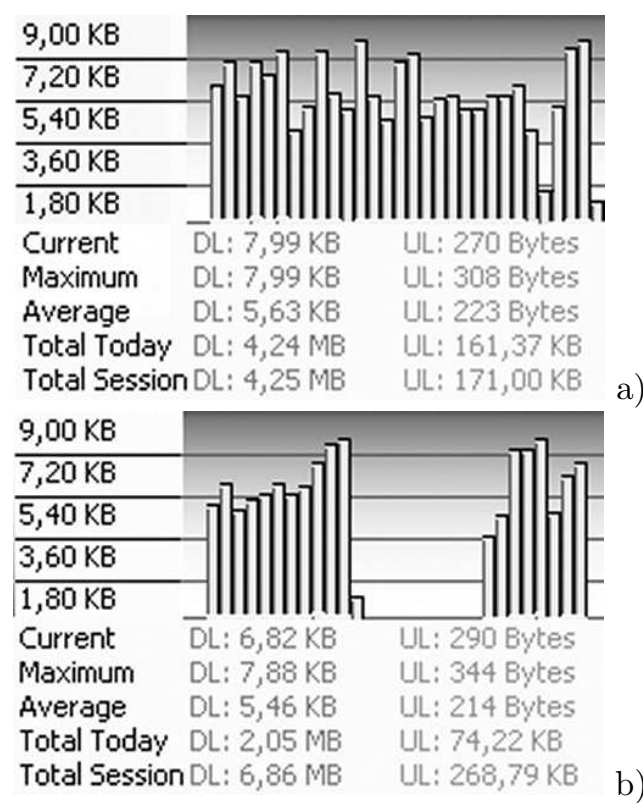

Fig. 10. Monitoring VPN/IP

\subsection{Connection by VPN/IP on ISDN}

Videos have been sent with an interval of one hour throughout different days and samples have been taken every two seconds (see Fig.10). A grater speed variation has been observed than in the point to point connection (see Fig.10a), since the speed of the connection depends on: the speed that the ISP (Internet Service Provider) offers and the state of the Internet. In Fig.10b, it is possible to observe that there are moments at which the communication is interrupted and then reinitiated after a few seconds. This creates a decrease in the average value of the speed of the channel and its reliability

Owing to the three sequences having similar image sizes, an average has been used to calculate the image per second rates in different hour strips for the different grades of compression.

As can be seen in Fig.11, for each of the compression grades the number of images/sec which can be transmitted is always smaller than with the point to point connection. There are also reliability problems that can cause the instability in the channel speed.

\section{CONCLUSIONS}

With the proposed new system, the state and alarms of each of the remote stations is obtained simultaneously since it is now unnecessary to wait for any polling cycle. The system has a greater immunity against radioelectric interference which increases its safety and reliability. The new network provides an improvement in many aspects, keeping the polling system as a backup system in 


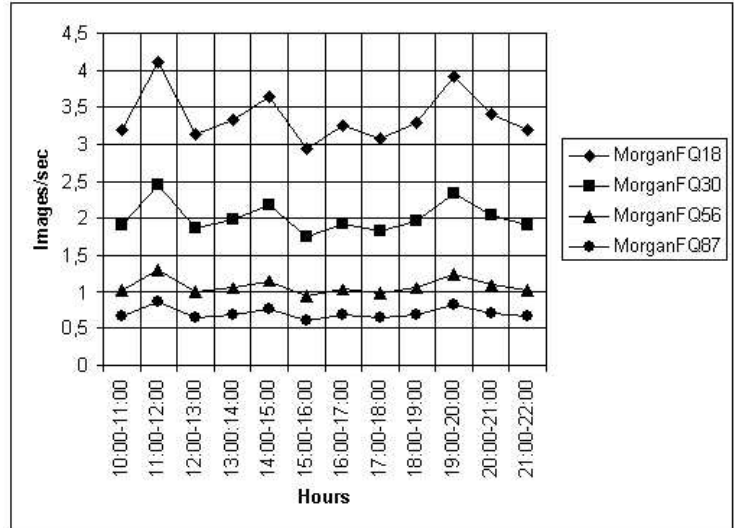

Fig. 11. Images/sec in the connection by VPN/IP, for different levels of compression

the case of failure in the public communication network.

After a carrying out the experiments the chosen solution for communication between the central station and the remote stations is point to point connection through RDSI. A greater number of images can be transmitted at a constant speed while with the VPN/IP a great variation in speed and even cuts in transmission have been observed, which is not advisable in remote control due to cuts in transmission being unacceptable in cases of emergency. It would be necessary to negotiate a determined quality of connection with the service provider to implement the use of this solution.

For the case of remote consultation through the Internet, the great flexibility from a communication point of view make this a powerful tool for the supervisors of large installations since the sending and receiving of critical information in certain incidences is now possible through various solutions such as mobile telephones and PDA's with adequate time responses. In this case, the tested solution of VPN/IP is perfectly applicable given that the consulting of information is limited.

\section{REFERENCES}

Ferguson, P. and G. Huston (1998). Quality of Service: Delivering QoS on the Internet and in Corporate Networks. Wiley.

Haverkort, B.R. (1999). Performance evaluation of polling based communication systems using spns. In: Application of Petri Nets to Communication Networks: Advances in Petri Nets (J. Billington, M. Diaz and G. Rozenberg, Eds.). 1rd ed.. pp. 176-209. Springer. Berlin.

Hoymann, C. and P. Stuckmann (2002). On the feasibility of video streaming applications over gprs/egprs. IEEE Global Telecommunications Conference.

Ibe, O.C. and K.S. Trivedi (1990). Stochastic petri net models of polling systems. IEEE
Journal on Selected areas in Communications 8(9), 1649-1657.

Kosiur, D. (1998). Building and Managing Virtual Private Network. Wiley.

Luque, J., J.I. Escudero and I. Gomez (1996). Determinig the channel capacity in scada systems using polling protocols. IEEE Transactions on Power Systems 11(2), 917-922.

Woods, D. (2002). Shriking the video: How codecs works. Network Computing pp. 77-79. 